\title{
Non-Microsurgical Technique of Fingertip Replantation: A Report of Three Cases
}

\author{
Emmanuel P. Estrella1,
}

\begin{abstract}
${ }^{1}$ Microsurgery Unit, Department of Orthopedics, College of Medicine and Philippine General Hospital, University of the Philippines Manila ${ }^{2}$ ASTRO (Advanced STudy and Research in Orthopedics) Study Group, National Institutes of Health, University of the Philippines Manila
\end{abstract}

\begin{abstract}
The goal of surgery in fingertip amputation is to restore finger length, preserve function and at the same time provide cosmetic acceptability. Treatment options are varied and can range from simple suturing of the stump to microvascular replantation surgery. We report three cases of fingertip amputations in one adult and two pediatric patients treated with non-microsurgical replantation of the fingertip using the palmar "pocket" technique.
\end{abstract}

Key Words: fingertip amputation, non-microsurgical replantation, finger replantation

\section{Introduction}

Fingertip amputations are challenging to treat. A variety of options from non-adherent dressings to complex microsurgical replantation exist. Microsurgical replantation is difficult because both terminal arteries and veins are small, especially in children. Alternatives to microsurgical replantation include non-operative treatment with daily dressing changes, skin grafts, local advancement flaps, and cross-finger flaps, and non-microsurgical composite grafting. ${ }^{1-7}$

Several reports on the pocketing technique of nonmicrosurgical replantation of fingertip amputations have had conflicting results. In the 1979 study by Brent, ${ }^{8}$ of the four fingers replanted using this procedure, only two fingers were viable. In 2001, Arata et al. ${ }^{9}$ reported 16 palmar pocket techniques for fingertip amputations where 13 of the patients were adults and 3 were children. The results of their study showed partial necrosis in three fingers ( 2 in adults and 1 in a child). Muneuchi et al. ${ }^{10}$ in 2005 reported subcutaneous pocketing in replanted fingertip amputations (five fingers in the ipsilateral palm and one in the abdomen). Only one survived completely but became atrophic after 4 months. One finger developed necrosis involving less than

Corresponding author: Emmanuel P. Estrella, MD

Microsurgery Unit, Department of Orthopedics

Philippine General Hospital

University of the Philippines Manila

Taft Avenue, Ermita, Manila 1000 Philippines

Telephone: +6325548466

Email: estee96@yahoo.com half of the replant but a hooked nail deformity developed. Two fingers developed partial necrosis involving more than half of the replant. Three fingers developed total necrosis. In four patients, a slight flexion contracture developed. Indications for the "palmar pocket" include cases where microsurgical replantation is not deemed feasible because of the size of the vessels and increasing the vascular supply of the amputated part if the patency of the vascular anastomosis during replantation surgery is suspect. Contraindications include cases where the amputation level is proximal to the distal interphalangeal joint, and possible thumb amputations. ${ }^{8,9}$

We report three cases of non-microsurgical replantation of fingertip amputations zone III according to Allen ${ }^{11}$ (Figure 1) using the palmar pocket technique.

\section{Case Reports}

Three patients had the "palmar pocket" technique for fingertip amputation. Informed consent was taken from the patient and patients' parents (in the pediatric population) with regard to inclusion in this study. The first patient was a 12-year-old girl (Patient 1) who sustained a fingertip amputation Allen Zone type III of the right ring finger (Figure 2A and 2B). The second patient was a 15-year-old boy (Patient 2) with a fingertip amputation of the right index finger Allen Zone type III (Figure 2C and 2D). The third patient was a 26-year-old male (Patient 3) who sustained a fingertip amputation Allen III of the middle finger of the right hand (Figure $2 \mathrm{E}$ and $2 \mathrm{~F}$ ). On all three occasions, the crushing injury was caused by a closing door. There was no fracture of the tuft on all three amputations.

The palmar pocket technique was used to replant the amputated fingertips. Once microsurgical anastomosis was deemed not feasible, the amputated stump was debrided and the subcutaneous fat was removed. De-epithelialization of the amputated part was done to expose the dermal layer. The amputated stump was then debrided and prepared for replantation. The amputated fingertip was then sutured to the stump using nylon 6-0 sutures and the nailbed was repaired using nylon 8-0 sutures (Figure 3). A 2-cm incision was then made on the palmar area, incising the palmar fascia. The finger was then "pocketed" up to the distal interphalangeal crease deep to the palmar fascia and this 
was held to the palmar skin with nylon 4-0 sutures (Figure 4). The wrists were then immobilized in slight extension and a dorsal blocking splint was made on the digits. The "pocketed" finger was maintained for 21 days after which the finger was released under local anesthesia. Proximal interphalangeal joint stiffness was evident for up to two weeks post-release and resolved within 3-4 weeks. Active range of motion exercises were encouraged on both cases and there was no need for a formal physical therapy referral. At four months post-release, Patient 1 had excellent range of motion of the interphalangeal joints and no residual nail deformity (Figure 5). There was no painful neuroma on the fingertip, static 2-point discrimination test was $5 \mathrm{~mm}$ and the Semmes-Weinstein filament number was 2.83 or 0.07 grams force.

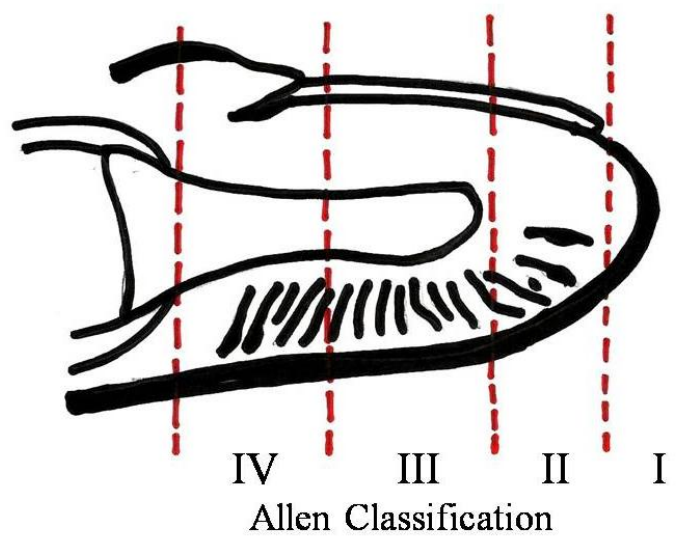

Figure 1. The classification of fingertip amputation according to Allen. ${ }^{11}$

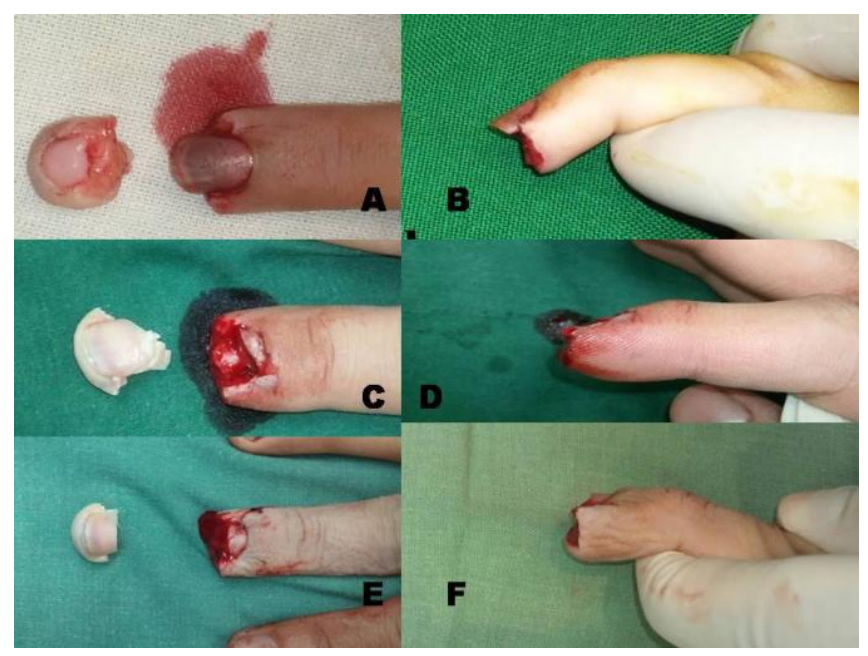

Figure 2. Dorsal and lateral views of fingertip amputations in Patient 1 (A and B), Patient 2 (C and D) and Patient 3 (E and F).

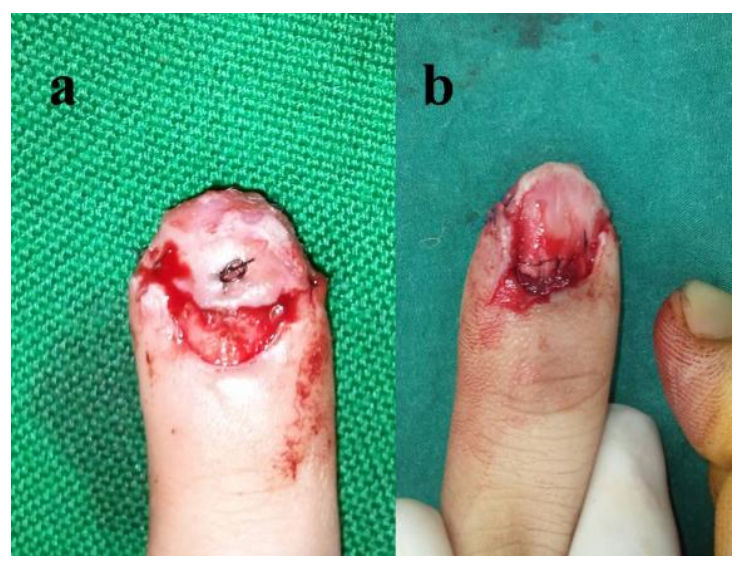

Figure 3. After the amputated stumps were "deepithelialized", they were replanted in the proximal stump with fine sutures. Patient 1 (a) and Patient 2 (b).

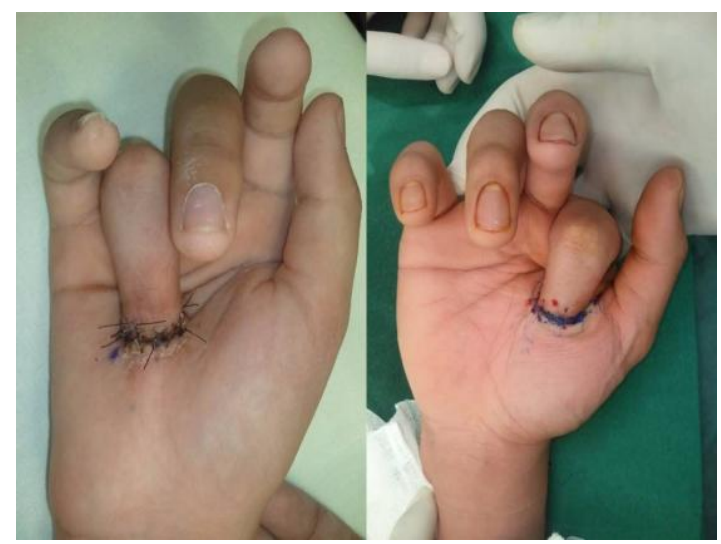

Figure 4. After replanting the fingertips, an incision was made on the palmar aspect of the same hand and the replanted fingertips were buried up for 3 weeks for Patient 1 (left) and Patient 2 (right).

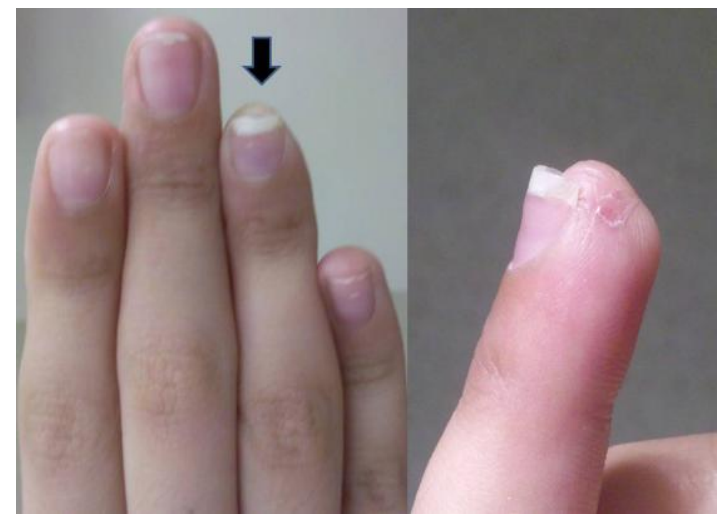

Figure 5. On latest follow-up at 4 months after the release from the palmar-pocket procedure, Patient 1 had normallooking nailgrowth and restoration of length of the ring finger (black arrow). On the right, atrophy of the replanted tip was inconspicuous. 
Patient 2 on latest follow-up at 10 months post-release had full range of motion of the interphalangeal joints with very minimal nail deformity (Figure 6). There was no painful neuroma of the fingertip and the static 2-point discrimination was $5 \mathrm{~mm}$, with a Semmes-Weinstein filament test of 2.83 or 0.07 grams force.

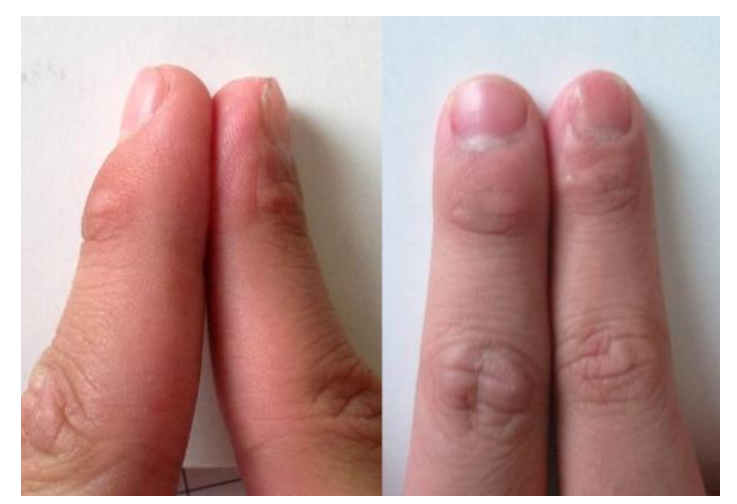

Figure 6. Patient 2 at 10 months post release of the palmarpocket produre. On the right picture, a dorsal view of sideto-side comparison of the replanted fingertip (on the right) and the normal index finger (on the left). Minimal nail deformity was evident on the replanted fingertip. On the left picture, side views of the left and right (replanted) index fingers showed adequate hyponichium and restoration of pulp mass.

Patient 3 on latest follow-up at 9 months post-release also had full range of motion with a slightly shorter nail compared to the opposite middle finger (Figure 7). However, the patient was very satisfied with the result. The static 2-point discrimination test was $6 \mathrm{~mm}$ and the SemmesWeinstein filament number was also 2.83 or 0.07 grams force. All patients report no functional limitation in the use of the involved hand or digit.

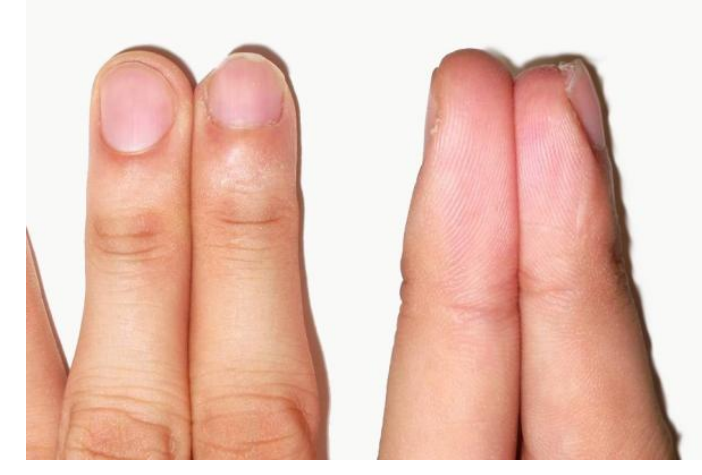

Figure 7. Patient 3 at 9 months post-release. The reconstructed fingertip is on the right. There is slight shortening of the fingernail of the reconstructed finger, however, the patient was very satisfied with the result.

\section{Discussion}

In fingertip amputations, success rates for microsurgical replantations range from $70-80 \% .{ }^{12}$ Replantation of the amputated part offers the restoration of the digit's original length, normal nail complex and an acceptable cosmetic appearance. However, because of the steep learning curve in microsurgical replantation surgery and the small size of the distal vessels, alternatives to microsurgical replantation with preservation of the amputated tip have been reported. These include composite grafting and composite grafting with pocketing technique procedures. Both composite grafting with and without pocketing technique rely on plasmatic imbibition for vascularity of the amputated stump.

Success rates for composite grafting without the pocketing technique range from $22 \%$ to $93.5 \% .^{13-16}$ Because vascularization of the composite graft occurs by plasmatic imbibition, increasing the surface area for vascular ingrowth theoretically increases the survival.

In 1979, Brent ${ }^{8}$ reported the "pocketing" technique after non-microsurgical replantation in the abdomen. Deepithelialization theoretically increases the surface area for vascular in-growth. Since then, other authors have reported variable success rates for the "pocketing" technique, ${ }^{17}$ with higher success rates reported in children. ${ }^{18}$ With regard to "pocketing", Brent ${ }^{8}$ proposed the "cross-chest" pocket, which is similar in position to the infraclavicular flap. Lee et al. ${ }^{17}$ proposed "pocketing" the amputated part in the abdomen, between the subcutaneous tissue and muscle to allow for a more vascularized tissue in muscle to be in contact with the amputated part. Arata et al. in $2001^{9}$ reported very good results in adults and children using the "pocketing" technique. This time, instead of "pocketing" the amputated part in the abdomen, it was pocketed in the ipsilateral palm to allow less disability of the upper extremity. However, complications of PIPJ stiffness and contractures were reported by subsequent authors using this technique, ${ }^{10,19}$ especially if performed in adults.

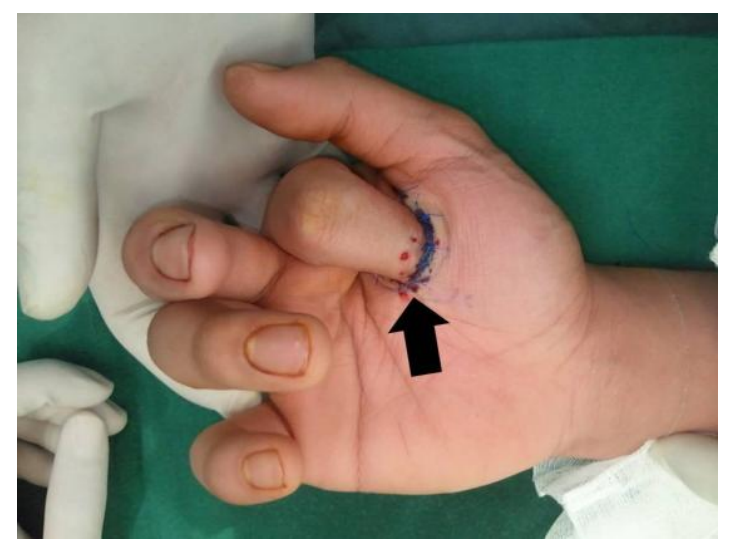

Figure 8. We modified the techniques of previous authors by burying not just the replanted part but by burying the finger up to the distal interphalangeal joint level (black arrow). 
We agree with Lee et al. ${ }^{17}$ that the critical steps in this technique should be appropriate de-epithelialization and adequate contact with the vascularized tissue. However, while they proposed abdominal pocketing, in our three cases, we used ipsilateral palmar pocketing technique. We made modifications from the initial report of Arata et al. in 2001.9 Instead of purely "subcutaneous" pocketing, we opened up the palmar fascia and pocketed the amputated tissue deep to the fascia. By doing this, we pocketed the tissue up to the DIPJ (distal interphalangeal joint), whereby the entire amputated stump was "pocketed" (Figure 8). By increasing the depth of the "pocketing", increased vascular contact can be assured and inadvertent pull-out of the finger may be avoided.

Although microsurgical anastomosis is still probably the best method to replant fingertip amputations, we suggest this technique in fingertip amputations up to Allen type III if microsurgical anastomosis is not feasible, especially in centers where adequate staff and equipment are not available, and if the patency of the initial vascular anastomosis is in question.

In summary, non-microsurgical replantation of fingertip amputation is possible with the palmar "pocket" technique. This technique has the potential to preserve normal finger length and normal nail contour and cosmesis. Selection of the level of amputation whereby this technique will be applied may need careful consideration. This technique may be ideal for more distal amputations (Allen Types I-III).

\section{References}

1. Yeo CJ, Sebastin SJ, Chong AK. Fingertip injuries. Singapore Med J. 2010; 51(1):78-87.

2. Mennen $U$, Wiese A. Fingertip injuries management with semi-occlusive dressing. J Hand Surg Br. 1993; 18(4):416-22.

3. Russell RC, Casas LA. Management of fingertip injuries. Clin Plast Surg. 1989; 16(3):405-25.

4. Atasoy E, Ioakimidis E, Kasdan ML, Kutz JE, Kleinert HE. Reconstruction of the amputated fingertip with a triangular volar flap. J Bone Joint Surg. 1970; 52(5):921-6.

5. Kutler W. A new method for the fingertip amputation. J Am Med Assoc. 1947; 133:29.

6. Moberg E. Aspects of sensation in reconstructive surgery of the upper extremity. J Bone Joint Surg Am. 1964; 46:817-25.

7. Uysal A, Kankaya Y, Ulusoy MG, et al. An alternative technique for microsurgically unreplantable fingertip amputations. Ann Plast Surg. 2006; 57(5):545-51.

8. Brent B. Replantation of amputated distal phalangeal parts of fingers without vascular anastomoses, using subcutaneous pockets. Plast Reconstr Surg. 1979; 63(1):1-8.

9. Arata J, Ishikawa K, Soeda H, Sawabe K, Kokoroishi R, Togo T. The palmar pocket method: an adjunct to the management of zone I and II fingertip amputations. J Hand Surg Am. 2001; 26(5):945-50.

10. Muneuchi G, Kurokawa M, Igawa K, Hamamoto $\mathrm{Y}$, Igawa $\mathrm{HH}$. Nonmicrosurgical replantation using a subcutaneous pocket for salvage of the amputated fingertip. J Hand Surg Am. 2005; 30(3):562-5.

11. Allen MJ. Conservative management of finger tip injuries in adults. Hand. 1980; 12(3):257-65.

12. Hattori Y, Doi K, Sakamoto S, Yamasaki H, Wahegaonkar A, Addosooki A. Fingertip replantation. J Hand Surg Am. 2007; 32(4):548-55.
13. Moiemen NS, Elliot D. Composite graft replacement of digital tips. 2. A study in children. J Hand Surg Br. 1997; 22(3):346-52.

14. Uysal A, Kankaya Y, Ulusoy MG, et al. An alternative technique for microsurgically unreplantable fingertip amputations. Ann Plast Surg. 2006; 57(5):545-51.

15. Eo S, Hur G, Cho S, Azari KK. Successful composite graft for fingertip amputations using ice-cooling and lipo-prostaglandin E1. J Plast Reconstr Aesthet Surg. 2009; 62(6):764-70

16. Chen SY, Wang CH, Fu JP, Chang SC, Chen SG. Composite grafting for traumatic fingertip amputation in adults: technique reinforcement and experience in 31 digits. J Trauma. 2011; 70(1):148-53.

17. Lee PK, Ahn ST, Lim P. Replantation of fingertip amputation by using the pocket principle in adults. Plast Reconstr Surg. 1999; 103(5):1428 -35.

18. Arata J, Ishikawa K, Soeda H. Replantation of fingertip amputation by palmar pocket method in children. Plast Reconstr Surg. 2011; 127(3):78e$80 \mathrm{e}$.

19. van Nieuwenhoven CA, van der Lei B, van Wingerden JJ. Nonmicrosurgical Replantation Using a Subcutaneous Pocket for Salvage of the Amputated Fingertip. J Hand Surg Am. 2006; 31(1):152. 